This item was submitted to Loughborough's Research Repository by the author.

Items in Figshare are protected by copyright, with all rights reserved, unless otherwise indicated.

\title{
Fast Fourier virtual fields method for determination of modulus distributions from full-field optical strain data
}

PLEASE CITE THE PUBLISHED VERSION

http://dx.doi.org/10.1007/978-3-642-36359-7_21

\section{PUBLISHER}

(c) Springer-Verlag Berlin Heidelberg

VERSION

AM (Accepted Manuscript)

\section{PUBLISHER STATEMENT}

This work is made available according to the conditions of the Creative Commons Attribution-NonCommercialNoDerivatives 4.0 International (CC BY-NC-ND 4.0) licence. Full details of this licence are available at: https://creativecommons.org/licenses/by-nc-nd/4.0/

\section{LICENCE}

CC BY-NC-ND 4.0

\section{REPOSITORY RECORD}

Nguyen, Truong Tho, Jonathan M. Huntley, lan A. Ashcroft, Pablo D. Ruiz, and Fabrice Pierron. 2019. "Fast Fourier Virtual Fields Method for Determination of Modulus Distributions from Full-field Optical Strain Data". figshare. https://hdl.handle.net/2134/14043. 


\title{
Fast Fourier Virtual Fields Method for Determination of Modulus Distributions from Full-field Optical Strain Data
}

\author{
Truong Tho Nguyen ${ }^{1}$, Jonathan M. Huntley ${ }^{1}$, Ian A. Asheroft ${ }^{2}$, \\ Pablo D. Ruiz ${ }^{1}$ and Fabrice Pierron ${ }^{3}$ \\ ${ }^{1}$ Loughborough University, Wolfson School of Mechanical and Manufacturing Engineering, \\ Loughborough LE11 3 TU, UK \\ ${ }^{2}$ Nottingham University, Faculty of Engineering, University Park, Nottingham NG7 2RD, \\ UK \\ ${ }^{3}$ University of Southampton, Faculty of Engineering and the Environment, Highfield, \\ Southampton SO17 1BJ, UK
}

\section{Introduction}

Inspection of parts for manufacturing defects or in-service damage is often carried out by full-field optical techniques (e.g., digital speckle pattern interferometry, digital holography) where the high sensitivity allows small anomalies in a loadinduced deformation field to be measured. Standard phase shifting and phase unwrapping algorithms provide full-field displacement and hence strain data over the surface of the sample. The problem remains however of how to quantify the spatial variations in modulus due, for example, to porosity or damage-induced microcracking. Finite element model updating (FEMU) is one method to solve problems of this type, by adjusting an approximate finite element model until the responses it produces are as close to those acquired from experiments as possible.

An alternative approach is the Virtual Fields Method (VFM) [1]. The advantage of the VFM over other methods is its ability to solve inverse problems of this type without iterative computation. Several approaches based on polynomial virtual fields with the material properties considered as to be single valued within the domain have been developed [2-5]. The first attempt to parameterize the material properties as a function of spatial variables was proposed in [6].

In this paper, we retain the basic concepts of the VFM but approach the parameterization of the material properties in the spatial frequency, rather than spatial, domain by performing a 2-D Fourier series expansion of the stiffness distribution over the region of interest. Furthermore, the virtual fields are not selected as polynomials of spatial variables as in the previous VFM literature, but from a set of simple cosine or sine functions of different spatial frequencies. This Fourier version of the VFM will be denoted the F-VFM. An example of its successful application to the identification of an unknown stiffness distribution under known boundary conditions is summarized here; further details are given in [7]. 


\section{Theoretical}

\subsection{Virtual Fields Method formulation}

For a thin 2-D sample made of an isotropic material, subject to known traction distributions around its boundary and negligible volume forces, the fundamental equation underlying the VFM may be written [1,7]:

$$
\int_{S}\left(\left(\varepsilon_{x x}+v \varepsilon_{y y}\right) \varepsilon_{x x}^{*}+\left(\varepsilon_{y y}+v \varepsilon_{x x}\right) \varepsilon_{y y}^{*}+\frac{1-v}{2} \varepsilon_{s s}^{*} \varepsilon_{s s}\right) Q_{x x} d S=\int_{\ell}\left(T_{x} u_{x}^{*}+T_{y} u_{y}^{*}\right) d \ell
$$

where $S$ is the area of interest over which the experimental data are available; $\ell$ is the part of the boundary of $S$ on which tractions exist; $\varepsilon_{x x}$ and $\varepsilon_{y y}$ are the normal strains as measured along the $x$-and $y$-axes of a Cartesian coordinate system, $\varepsilon_{s s}$ is the engineering shear strain; $\left(T_{x}, T_{y}\right)$ are the components of the traction vector defined over $\ell ; u_{x}^{*}$ and $u_{y}^{*}$ are the components of the virtual displacement field with $\varepsilon_{x x}^{*}, \varepsilon_{y y}^{*}$ and $\varepsilon_{s s}^{*}$ the corresponding virtual strain field components. The unknown stiffness distribution $Q_{x x}(x, y)$ is related to Young's modulus and Poisson's ratio $v$ (assumed to be a constant) by $Q_{x x}=E /\left(1-v^{2}\right)$.

\subsection{Fourier series expansion of stiffness distribution}

The stiffness distribution $Q_{x x}(x, y)$ in Eq. 1. may be expanded as a 2-D Fourier series and written in matrix form as follows:

$$
Q_{x x}(x, y)=\left(\begin{array}{llll}
1 & \ldots \mathcal{c}_{m, n}(x, y) \ldots & \ldots s_{m, n}(x, y) \ldots
\end{array}\right)\left(\begin{array}{c}
a_{0,0} \\
\vdots \\
a_{m, n} \\
\vdots \\
b_{m, n} \\
\vdots
\end{array}\right)
$$

where cosine and sine functions with non-dimensional spatial frequency components $(m, n)$, where $m, n=0,1,2, \ldots, N$, are represented by the shorthand notation 
$c_{m, n}(x, y)=\cos 2 \pi\left(m x / L_{x}+n y / L_{y}\right)$ and $s_{m, n}(x, y)=\sin 2 \pi\left(m x / L_{x}+n y / L_{y}\right)$, respectively. The column vector on the right hand side of Eq. 2. is the sought solution vector, consisting of $(N+1)^{2} \quad a_{m, n}$ coefficients associated with the cosine functions and $(N+1)^{2}-1 \quad b_{m, n}$ coefficients associated with the sine functions. The total number of degrees of freedom in the identification problem is therefore

$$
N_{F}=2(N+1)^{2}-1
$$

\subsection{Selection of virtual fields in the F-VFM}

The natural choice for the virtual fields in the F-VFM is an arrangement of simple cosine and sine functions. Eq. 1. involves area integrals of terms of the form $\varepsilon_{\alpha \alpha}^{*} \varepsilon_{\beta \beta} Q_{x x}(\alpha, \beta=x, y, s)$; the use of different spatial frequencies in the virtual fields therefore allows a given spatial frequency in the measured strain field $\varepsilon_{\beta \beta}$ to be linked in turn with different coefficients in the $Q_{x x}$ expansion.

The approach taken here was to choose the $\varepsilon_{x x}^{*}$ and $\varepsilon_{y y}^{*}$ fields to consist of a set of cosine waves (with spatial frequency components $(p, q)$ where $p, q=$ $0,1, \ldots, N$, giving $(N+1)^{2}$ independent virtual fields), and a set of corresponding sine waves (in which the trivial case $p=q=0$ is excluded, giving an additional $(N+1)^{2}$ - 1 fields). The total number of chosen cosine and sine virtual fields will therefore be equal to $N_{F}$, which is the required number to determine uniquely the unknown Fourier series coefficients of Eq. 2.

Substituting these virtual fields into Eq. 1. results in the matrix equation:

$$
\mathbf{M X}=\mathbf{Y}
$$

where $\mathbf{M}$ is an $N_{F} \times N_{F}$ matrix whose elements are calculated from the experimental strain fields, $\mathbf{X}$ is the desired $N_{F} \times 1$ solution vector of Fourier coefficients, and $\mathbf{Y}$ is an $N_{F} \times 1$ column vector calculated from the tractions. Eq. 4 can be inverted by the MATLAB 'pinv' function.

\subsection{Fast Fourier VFM implementation}

When applying Eq. 1. to experimental data, the measured strain fields $\varepsilon_{x x}, \varepsilon_{y y}$ and $\varepsilon_{s s}$ are normally sampled on a regular grid and the integrals are replaced by 
summations. If the experimental strain fields have $N_{x} \times N_{y}$ pixels, then a single contributory term to one of the elements of $\mathbf{M}$ requires a minimum of $N_{x} N_{y}$ addition plus multiplication operations. The computational effort to calculate $\mathbf{M}$ therefore scales as $N_{F}^{2} N_{x} N_{y}$. For example, the application in the next section, with $N_{x}$ $=N_{y}=1000$ and $N_{F}=881(N=20)$, took approximately $5.5 \times 10^{3}$ s to set up the matrix $\mathbf{M}$ on an Intel ${ }^{\circledR}$ Core $^{\mathrm{TM}}$ i7 $\mathrm{CPU} 2.79 \mathrm{GHz}$ machine with $8 \mathrm{~GB}$ of memory.

A much more efficient algorithm can be implemented, however, using 2-D fast Fourier transforms. The fact that both the expansion of $Q_{x x}$ and the virtual fields are represented as sine and cosine functions means that the integrals can be expressed as 2-D Fourier coefficients of a linear combination of the experimental strain fields. It can be shown [7] that a total of only four 2-D Fast Fourier Transforms (FFTs) are required to assemble all the terms in M. For large matrix sizes, the computational effort becomes essentially independent of the resolution of the experimental strain fields, with a theoretical reduction in computational effort by a factor of $N_{x} N_{y}$ by using the fast algorithm over the direct (i.e., element by element) method of assembling the matrix $\mathbf{M}$.

The computation time for the other steps in the algorithm, i.e. evaluation of the terms in the vector $\mathbf{Y}$; the inversion of Eq. 4.; and reconstruction of the elastic stiffness distribution from the solution vector, is normally short compared to that for calculation of $\mathbf{M}$. The reconstruction can be handled very efficiently by performing a single 2-D inverse Fourier transform on a 2-D array of complex numbers that is obtained directly from $\mathbf{X}$.

For the problem considered in the next section, the total calculation time for the stiffness identification using the fast algorithm when implemented as a MATLAB script, on an Intel® Core ${ }^{\mathrm{TM}}$ i7 CPU $2.79 \mathrm{GHz}$ machine with 8GB of memory, was $\sim 2.5 \mathrm{~s}$ and $250 \mathrm{~s}$ for problem sizes $N=20$ and $N=80$, respectively. This may be compared with values of $6.1 \times 10^{3} \mathrm{~s}$ and $3.7 \times 10^{6} \mathrm{~s}$, respectively, for the direct method. A time saving of 3-4 orders of magnitude is therefore clearly achievable in practice.

\section{Example application}

In this section we give proof-of-principle results from the F-VFM method presented above with a complex stiffness distribution under uniform loading conditions. The input data to the F-VFM method were provided by a forward calculation from known stiffness distributions by the finite element (FE) method, thus providing a benchmark to compare the reconstructed stiffness maps against. The FE model used to generate the input strain fields consisted of a thin square plate of size $L_{x} \times L_{y}=10 \times 10 \mathrm{~mm}^{2}$ and of thickness $t=1 \mathrm{~mm}$. The geometry was meshed in Mentat2010 using $1000 \times 1000$ linear quadrilateral elements with full integration. Two vertical edges of the plate perpendicular to the $x$-axis were loaded with a uniformly distributed stress $\sigma_{x x}=1 \mathrm{MPa}$ pointing outwards. The origin of the coordi- 
nate system is at the centre of the plate. The material was chosen to be linear elastic isotropic with the reference elastic modulus distribution given by an 'egg-box' pattern of spatially varying stiffness distribution:

$$
E=20+\cos 2 \pi\left(\frac{2 x}{L_{x}}+\frac{y}{L_{y}}\right)+\sin 2 \pi\left(\frac{x}{L_{x}}+\frac{2 y}{L_{y}}\right)
$$

and a constant Poisson's ratio $v=0.3$. Plane stress conditions are applicable in this case since the plate thickness is small compared to the other dimensions.

Some of the main results are shown in Fig. 1. Ripples in the recovered stiffness map are observed but can be largely removed by smoothing with a uniform square kernel of size equal to the pitch $p$ (= 50 pixels $)$ of the highest frequency fringes. The edge effect region of 25 pixels wide (half of the kernel window size) resulting from the convolution is masked out from the reconstructed stiffness as in Fig. 1(c). The residual in the error map (Fig. 1(d)) represents a difference of about $0.5 \%$ between the reference and recovered stiffness distributions.

\section{Conclusions}

The paper presents a development of the virtual fields method by implementing a novel parameterization of the stiffness distribution with a full 2-D Fourier series expansion. An example stiffness distribution has been reconstructed after a single computation step without any iteration. A highly efficient numerical algorithm based on the fast Fourier Transform allows an identification problem with $\sim 10^{3}$ degrees of freedom to be solved in just a few seconds. The spatial resolution of the recovered stiffness by F-VFM is directly controllable through the choice of maximum spatial frequency. In this study the reconstructed modulus fields were obtained under the assumption that the traction distributions are known over the boundaries. In the future the F-VFM will be extended to cope with the cases where boundary conditions are unspecified over at least a part of the boundary of the domain of interest. Other important remaining issues are application of the method to the case of anisotropic materials such as carbon-fiber reinforced composites.

\section{References}

1. Pierron, F, Grédiac, M(2012) The Virtual Fields Method: Extracting constitutive mechanical parameters from full-field deformation measurements. Springer, New York

2. Pierron, F, Grédiac, M (2012) T-shaped specimen for the direct characterization of orthotropic materials. International Journal for Numerical Methods in Engineering 41:293309 
(a)

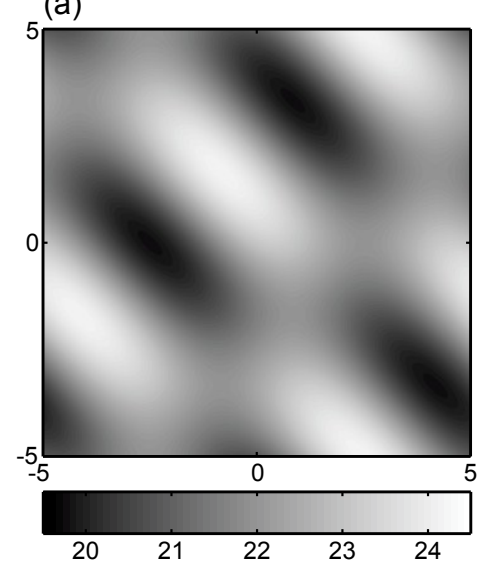

(c)

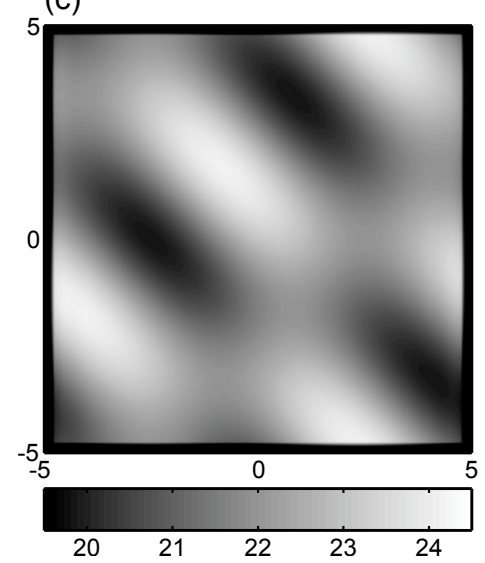

(b)

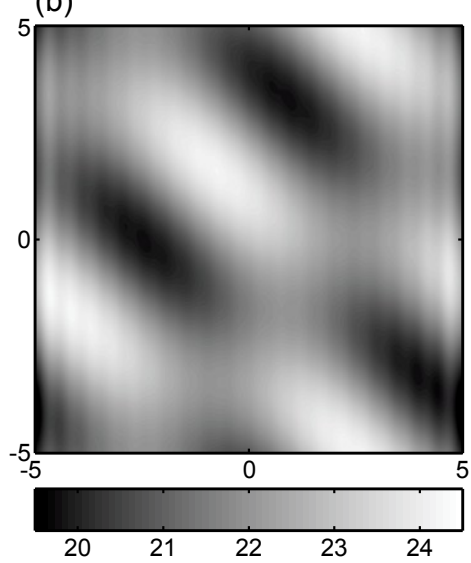

(d)

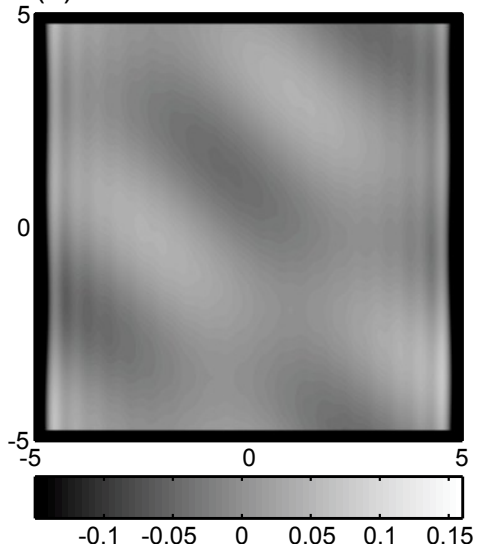

Fig. 1. Recovery of the 'egg-box' stiffness distribution by the F-VFM with $N=20$ ( 881 degrees of freedom, units: MPa). (a) Reference stiffness map (1000×1000 pixels); (b) Recovered stiffness map by F-VFM; (c) as (b) after smoothing by a $50 \times 50$ pixel kernel; (d) Error map

3. Grédiac, M, Toussaint, E, Pierron, F (2002) Special virtual fields for the direct determination of material parameters with the virtual fields method. 1 - Principle and definition. International Journal of Solids and Structures 39:2691-2705

4. Avril, S, Grédiac, M, Pierron, F (2004) Sensitivity of the virtual fields method to noisy data. Computational Mechanics 34:439-452

5. Toussaint, E, Grédiac, M, Pierron, F (2006) The virtual fields method with piecewise virtual fields. International Journal of Mechanical Sciences 48:256-264

6. Kim, JH, Pierron, F, Wisnom, MR, Syed-Muhamad, K (2007) Identification of the local stiffness reduction of a damaged composite plate using the virtual fields method. Composites Part A-Applied Science and Manufacturing 38:2065-2075

7. Nguyen, TT, Huntley, JM, Ashcroft, IA, Ruiz, PD, Pierron, F (2012) A Fourier-series-based Virtual Fields Method for the identification of 2-D stiffness distributions. International Journal for Numerical Methods in Engineering (under review) 